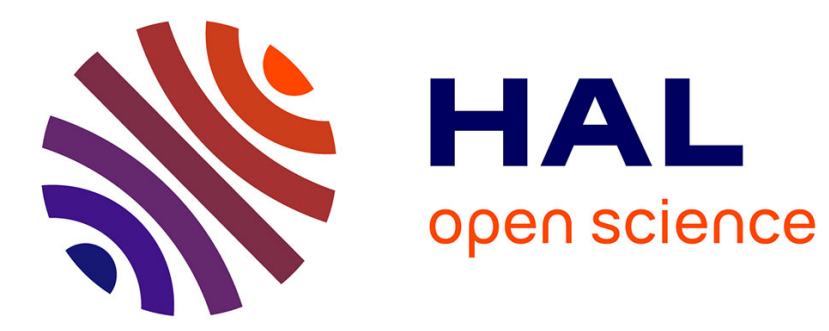

\title{
Torque approach for tuning exchange bias training effect in polycrystalline $\mathrm{NiFe} / \mathrm{FeMn}$ bilayers
}

\author{
Z. Shi, J. Du, R. W Chantrell, S. Mangin, S. M Zhou
}

\section{To cite this version:}

Z. Shi, J. Du, R. W Chantrell, S. Mangin, S. M Zhou. Torque approach for tuning exchange bias training effect in polycrystalline NiFe/FeMn bilayers. Applied Physics Letters, 2011, 98 (12), pp.122507. 10.1063/1.3569140 . hal-02085981

\section{HAL Id: hal-02085981 \\ https://hal.univ-lorraine.fr/hal-02085981}

Submitted on 1 Apr 2019

HAL is a multi-disciplinary open access archive for the deposit and dissemination of scientific research documents, whether they are published or not. The documents may come from teaching and research institutions in France or abroad, or from public or private research centers.
L'archive ouverte pluridisciplinaire HAL, est destinée au dépôt et à la diffusion de documents scientifiques de niveau recherche, publiés ou non, émanant des établissements d'enseignement et de recherche français ou étrangers, des laboratoires publics ou privés. 


\title{
Torque approach for tuning exchange bias training effect in polycrystalline NiFe/FeMn bilayers
}

\author{
Z. Shi, ${ }^{1}$ J. Du, ${ }^{2}$ R. W. Chantrell, ${ }^{3}$ S. Mangin, ${ }^{4}$ and S. M. Zhou ${ }^{1, a)}$ \\ ${ }^{1}$ Department of Physics, Tongji University, Shanghai 200092, China \\ ${ }^{2}$ National Laboratory of Solid State Microstructures, Nanjing University, Nanjing 210093, China \\ ${ }^{3}$ Physics Department, The University of York, York YO10 5 DD, United Kingdom \\ ${ }^{4}$ Nancy University, Inst Jean Lamour, F-54506 Vandoeuvre Les Nancy, France
}

(Received 31 January 2011; accepted 1 March 2011; published online 25 March 2011)

\begin{abstract}
In order to tune the exchange bias training effect, a torque approach is proposed. Torque loops are performed between two consecutive hysteresis loops by clockwise and counterclockwise rotation of the sample within an angular range under an in-plane external magnetic field. For the angular range from $0^{\circ}$ to $180^{\circ}$, the coercivity $H_{C}$ and the exchange field $H_{E}$ are both reduced and the pinning direction $(\mathrm{PD})$ orientation is changed, exhibiting the first type training effect. For the angular range from $-20^{\circ}$ to $380^{\circ}, H_{C}$ and the PD orientation are almost unchanged although $H_{E}$ is reduced, exhibiting the second type training effect. (C) 2011 American Institute of Physics.
\end{abstract}

[doi:10.1063/1.3569140]

Exchange coupling at the interface of ferromagnetic (FM) and antiferromagnetic (AFM) layers can induce a unidirectional anisotropy in the FM layer. In the exchange bias (EB) effect, ${ }^{1}$ the center of the hysteresis loop shifts and the coercivity $\left(H_{C}\right)$ often increases. Since its discovery, extensive research work has been done due to not only the abundant physics properties but also to the potential applications in spintronics. ${ }^{1-3}$

Among various effects of $\mathrm{EB}$, training effect has been studied extensively in various FM/AFM, and ferrimagnet/ ferrimagnet, and FM/FM systems. ${ }^{4-19}$ In the training effect, $H_{C}$ and exchange field $\left(H_{E}\right)$ decease with the cycling number $n$. Abrupt changes in $H_{E}$ and $H_{C}$ occur between $n=1$ and $n$ $=2 .{ }^{10}$ For $n>2$, an empirical power law between EB $\left(H_{E}\right.$ and $\left.H_{C}\right)$ and $n$ occurs. ${ }^{4,7,8,11,15}$ With respect to the behaviors of the coercive fields, two major types of training effect have been observed. ${ }^{6,16}$ For the first one, the coercive field of the descent branch changes very much whereas that of the ascent branch almost does not change. For the second one, the changes in the coercive fields of the two branches are close to each other, which is observed in either in-plane and outof-plane EB systems. ${ }^{6,16,17} H_{C}$ almost does not change during cycling although $H_{E}$ changes gradually. Up to date no mechanism explains the difference between the two training types. Since the shrinkage of $H_{E}$ and $H_{C}$ is strongly related to the pinning direction (PD), where the magnetization is aligned with zero $H$ (the external magnetic field), the training effect can in principle be tuned by controlling the PD. In this letter, we show that the type of training effect can be tuned by a special torque training procedure in which the torque loop is measured between two consecutive hysteresis loops. By rotation $\theta_{H}$ (the angle between $\mathrm{H}$ and the initial PD) from $0^{\circ}$ to $180^{\circ}$ (from $-20^{\circ}$ to $380^{\circ}$ ) one can observe the first (the second) type of training effect. Furthermore, motion of AFM spins is mapped out during both the conventional training and the torque training procedures.

Two samples of substrate $/ \mathrm{Cu}(20 \mathrm{~nm}) / \mathrm{Ni}_{80} \mathrm{Fe}_{20}(=\mathrm{NiFe})$ $(6 \mathrm{~nm}) / \mathrm{Fe}_{50} \mathrm{Mn}_{50}(=\mathrm{FeMn})\left(t_{\mathrm{AFM}}\right) / \mathrm{Au}(11 \mathrm{~nm})$ with $t_{\mathrm{AFM}}$

${ }^{\text {a)} E l e c t r o n i c ~ m a i l: ~ s h i m i n g @ t o n g j i . e d u . c n . ~}$
$=11 \mathrm{~nm}$ and $20 \mathrm{~nm}$ were deposited on Si wafers by magnetron sputtering at ambient temperature. The base pressure of the system is $1.8 \times 10^{-5} \mathrm{~Pa}$ and the Ar pressure $0.5 \mathrm{~Pa}$ during deposition. The deposition rates of constituting layers are about $0.1 \mathrm{~nm} / \mathrm{s}$. A magnetic field of about $170 \mathrm{Oe}$ is set in the film plane to induce a unidirectional anisotropy during deposition. X-ray diffraction in the high angle region shows that constituent layers are of (111) preferred orientation. In-plane $M-H$ loops are measured using vibrating sample magnetometer with vector coil, in which the magnetic moment parallel $\left(m_{x}\right)$ and perpendicular $\left(m_{y}\right)$ to $H$ are recorded simultaneously. The PD can be identified by angular variation in $m_{x}$ and $m_{y}$ under zero $H$. When $m_{y}=0$ and $m_{x}$ takes the maximum value, $\theta_{H}=0$ is set. In the conventional approach, consecutive hysteresis loops were measured without break. In the torque approach, torque loops were performed between two consecutive hysteresis loops by clockwise and counterclockwise rotation of samples in a specific $H$. $\theta_{H}$ changes from $0^{\circ}$ to $180^{\circ}$ or from $-20^{\circ}$ to $380^{\circ}$. All the measurements are carried out at room temperature.

For the NiFe/FeMn $(11 \mathrm{~nm})$ sample, the initial PD and $\theta_{H}=0^{\circ}$ are first identified. Then 10 torque loops are performed within the angular region from $0^{\circ}$ to $180^{\circ}$ in a magnetic field of $1 \mathrm{kOe}$, followed by a hysteresis loop at $\theta_{H}$ $=0^{\circ}$. In comparison, the conventional training effect is measured for another sample at $\theta_{H}=0^{\circ}$. Figures 1(a) and 1(b) show the results. For the $n=1$ loop (solid line) in the conventional training effect and the loop after $n^{\prime}=10$ (dot line) torque loops, $H_{E} / H_{C}$ equals $93 / 89$ and $59 / 43$ in the unit of oersted, respectively. For the $n=1$ loop $m_{y}$ is almost equal to zero, indicating the collinear alignment of the unidirectional and the uniaxial anisotropic axes before the torque training, and appears after the torque training, indicating the noncollinear alignment. ${ }^{20}$ Figure $1(\mathrm{c})$ shows the $m_{y}$ versus $\theta_{H}$ curves under $H=0$ of the as-deposited state (solid line), after $n^{\prime}=10$ torque loops (dot line), and after $n=1$ hysteresis loop (dash line). The PD is rotated from $0^{\circ}$ to $24^{\circ}$ after the torque training procedure, which coincides with nonzero $m_{y}$ in Fig. 1(b). 

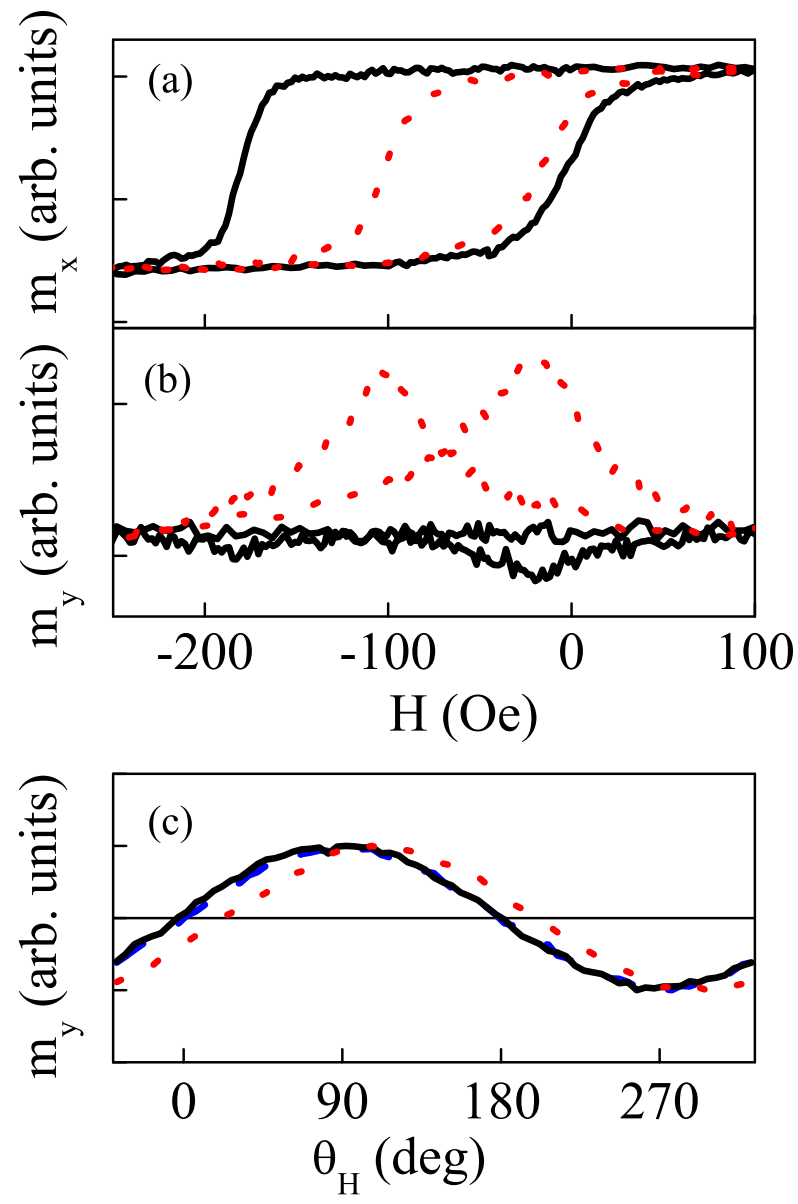

FIG. 1. (Color online) (a) $m_{x}$ and (b) $m_{y}$ vs $H$ of $n=1$ (solid line) in the conventional approach and after $n^{\prime}=10$ within the angular range from $0^{\circ}$ to $180^{\circ}$ (dot line). In (c) $m_{y}$ vs $\theta_{H}$ under $H=0$ at the as-prepared state (solid line), after ten torque loops (dot line), and after the $n=1$ hysteresis loop (dash line).

It is essential to reveal the effect of the angular rotation on the torque training effect. For the NiFe/FeMn (20 nm) sample, $n^{\prime}=20$ torque loops are measured within the angular region from $-20^{\circ}$ to $380^{\circ}$ and then hysteresis loop is measured at $\theta_{H}=0^{\circ}$. In comparison, the conventional training effect is also measured. Figures 2(a) and 2(b) show the results. $H_{E} / H_{C}$ equals $38.5 / 21.5$ Oe after $n^{\prime}=20$ and 46.5/20.5 Oe for $n=1$. Apparently $H_{E}$ is reduced whereas $H_{C}$ is almost unchanged and the reversal fields of the two branches is shifted by a similar amount. To obtain this second type of training effect the training torque procedure is needed. It is noted that the first type of training effect is caused by the conventional approach, ${ }^{6}$ as demonstrated by $n=1$ and $n=10$ hysteresis loops. Figure 2(c) shows that the PD orientation does not change significantly after both the torque training and the conventional consecutive training procedure when all hysteresis loops are measured at $\theta_{H}=0^{\circ}$. It shows that the torque loop performed between $-20^{\circ}$ and $380^{\circ}$ has no effect on the PD orientation. The angular rotation range is proved to have strong effects on the torque training effect.

To better understand the effect of the torque training procedure, it is essential to reveal the $n^{\prime}$ effect on the EB. Figure 3(a) shows the hysteresis loops of the NiFe/FeMn (11 $\mathrm{nm})$ bilayer after different $n^{\prime}$. Several features can be found. First of all, although $H_{E}$ decreases gradually from 93 to 74 Oe with increasing $n^{\prime}, H_{C}$ almost does not change. Second,
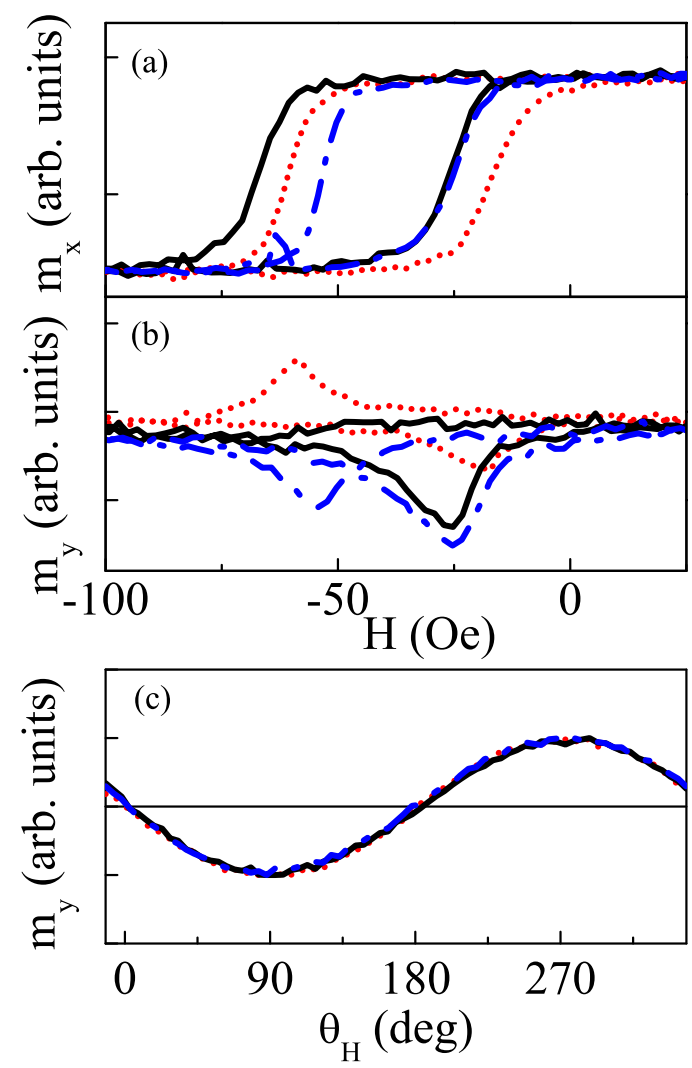

FIG. 2. (Color online) (a) $m_{x}$ and (b) $m_{y}$ vs $H$ for $n=1$ (solid line) and $n$ $=10$ (dash line) in the conventional approach and after $n^{\prime}=20$ within the angular region from $-20^{\circ}$ to $380^{\circ}$ (dot line). In (c) $m_{y}$ vs $\theta_{H}$ under $H=0$ at the as-prepared state (solid line), after $n^{\prime}=20$ within the angular region from $-20^{\circ}$ to $380^{\circ}$ (dot line), and $n=1$ (dash line) in the conventional approach.

the $m_{y}$ values of two branches are almost fixed, as shown in Fig. 3(b), which is different from the observed results in the conventional training effect. ${ }^{19}$ Moreover, as shown in Fig. $3(\mathrm{c})$, the PD is not rotated during the torque training procedure, which agrees with the behavior of $m_{y}$ as shown in Fig. 3(b). Furthermore, $H_{E}$ and $H_{C}$ depend on $n^{\prime}$, as shown in Fig. $3(d)$.

The behaviors of $H_{E}, H_{C}$, and the PD can be understood as follows. In AFM grains, AFM spins may be thermally stable or instable at finite temperatures, depending on the energy barrier which is equal to the product of the uniaxial anisotropy constant and the grain volume. ${ }^{21,22}$ In some stable AFM grains, AFM spins are fixed whereas AFM spins in other grains are rotated during the FM magnetization reversal process. Nonrotatable and rotatable grains, therefore, correspond to $H_{E}$ and $H_{C}$, respectively. During deposition under magnetic fields or magnetic field cooling procedure, the unidirectional and the uniaxial anisotropies are established. In the present $\mathrm{NiFe} / \mathrm{FeMn}$ bilayers, their axes are collinearly aligned at the as-prepared state as demonstrated by the fact that $m_{y}$ is always equal to zero when $\theta_{H}=0^{\circ} .^{20}$ During the training effects, the AFM spins in rotatable grains, the average orientation of AFM spins in all rotatable grains, and thus the uniaxial axis move to new equilibrium orientations. Similarly, the unidirectional axis may deviate from the initial PD. Therefore, the axes of the uniaxial anisotropy and the unidirectional one may be noncollinearly aligned and the PD orientation may be different from the initial one. At the same time, the magnitude of both the uniaxial and the unidirectional anisotropies may also be reduced. It is noted that the 

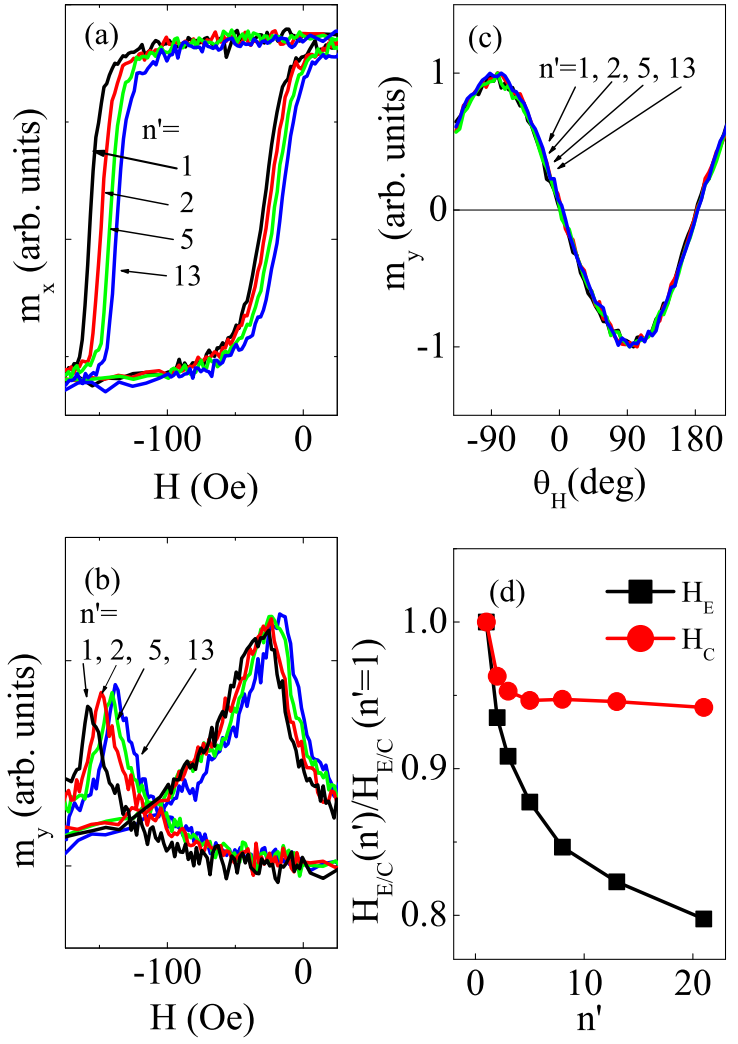

FIG. 3. (Color online) (a) $m_{x}$ and (b) $m_{y}$ vs $H$, and (c) $m_{y}$ vs $\theta_{H}$ under $H$ $=0$ with torque loops of $n^{\prime}=1,2,5$, and 13 within the angular region from $-20^{\circ}$ to $380^{\circ}$. In (d), the dependence of $H_{E}$ and $H_{C}$ on $n^{\prime}$.

rotation of AFM spins depends on the motion of the FM magnetization.

For the torque training procedure taking place between $0^{\circ}$ to $180^{\circ}$, the AFM spins in both rotatable and nonrotatable grains are deviated from the initial PD and orientated between $0^{\circ}$ to $180^{\circ}$, leading to the reduction in $H_{E}$ and $H_{C}$, and to the PD rotation. For the torque training procedure taking place between $-20^{\circ}$ and $380^{\circ}$, AFM spins in the rotatable grains are finally orientated along the initial PD after $\mathrm{CW}$ rotation from $-20^{\circ}$ and $380^{\circ}$ and subsequent $\mathrm{CCW}$ rotation from $380^{\circ}$ and $-20^{\circ}$, leading to the recovery of the axis and the magnitude of the uniaxial anisotropy. Meanwhile, the transition of AFM spins in the nonrotatable grains may still be triggered. Therefore, $H_{C}$ and the PD orientation are not changed much but $H_{E}$ is reduced. Similar results in the conventional training effects of $\mathrm{Pt} / \mathrm{Co} / \mathrm{Pt} / \mathrm{IrMn}$ multilayers and (110) epitaxially grown $\mathrm{NiFe} / \mathrm{FeMn}$ bilayer can be understood in the same way. ${ }^{16,17}$ For the $\mathrm{Pt} / \mathrm{Co} / \mathrm{Pt} / \mathrm{IrMn}$ multilayers with pinned domain wall motion, the magnetization of all domains is aligned along the film normal direction near coercive fields and thus the AFM spins can only be aligned along the film normal direction. Accordingly, the axis and the magnitude of the uniaxial anisotropy are not changed and the PD is not rotated either. Since AFM spins in nonrotatable grains may experience $180^{\circ}$ switching, $H_{E}$ is reduced. It should be noted that above discussion is only valid for poly- crystalline FM/AFM bilayers. For epitaxially grown FM/ AFM bilayers, new features occur in the EB training effect. For epitaxially grown FM/AFM bilayers, the EB training effect is much smaller than that of polycrystalline FM/AFM bilayers. ${ }^{16}$ The second type training effect occurs in epitaxially grown $\mathrm{NiFe} / \mathrm{FeMn}$ bilayers by conventional procedure possibly due to the smaller fraction of the rotatable AFM grains compared with that of polycrystalline ones.

In short summary, the torque training procedure is proposed to tune the EB training effect. When the angular rotation covers the $0^{\circ}$ to $180^{\circ}$ range, a first type training effect is induced, ${ }^{6}$ where $H_{C}$ and $H_{E}$ are both reduced, and the PD orientation changes. For the angular rotation range from $-20^{\circ}$ to $380^{\circ}$, a second type training effect is induced, ${ }^{6}$ where $H_{C}$ and the PD orientation are almost unchanged even so $H_{E}$ is reduced. Apparently, besides $H_{E}$ and $H_{C}$, the PD orientation is also of crucial importance to map out the motion of AFM spins during the training effect and to tune the EB training effect.

This work was supported by the National Natural Science Foundation of China under Grant Nos. 50871030, 11074112, and 10974032 and the National Basic Research Program of China under Grant No. 2009CB929201.

${ }^{1}$ W. H. Meiklejohn and C. P. Bean, Phys. Rev. 102, 1413 (1956).

${ }^{2}$ See, e.g., J. Nogués and I. K. Schuller, J. Magn. Magn. Mater. 192, 203 (1999).

${ }^{3}$ A. E. Berkowitz and K. Takano, J. Magn. Magn. Mater. 200, 552 (1999).

${ }^{4}$ D. Paccard, C. Schlenker, O. Massenet, R. Montmory, and A. Yelon, Phys. Status Solidi 16, 301 (1966)

${ }^{5}$ R. W. Chantrell, N. Walmsley, J. Gore, and M. Maylin, Phys. Rev. B 63, 024410 (2000).

${ }^{6}$ K. Zhang, T. Zhao, and M. Fujiwara, J. Appl. Phys. 89, 6910 (2001); 91, 6902 (2002).

${ }^{7}$ H. Xi, R. M. White, S. Mao, Z. Gao, Z. Yang, and E. Murdock, Phys. Rev. B 64, 184416 (2001).

${ }^{8}$ A. Hochstrat, C. Binek, and W. Kleemann, Phys. Rev. B 66, 092409 (2002).

${ }^{9}$ C. Binek, Phys. Rev. B 70, 014421 (2004).

${ }^{10}$ A. Hoffmann, Phys. Rev. Lett. 93, 097203 (2004).

${ }^{11}$ M. S. Lund and C. Leighton, Phys. Rev. B 76, 104433 (2007).

${ }^{12}$ S. Polisetty, S. Sahoo, and Ch. Binek, Phys. Rev. B 76, 184423 (2007).

${ }^{13}$ T. Hauet, S. Mangin, J. McCord, F. Montaigne, and E. E. Fullerton, Phys. Rev. B 76, 144423 (2007).

${ }^{14}$ Ch. Binek, S. Polisetty, X. He, and A. Berger, Phys. Rev. Lett. 96, 067201 (2006).

${ }^{15}$ M. K. Chan, J. S. Parker, P. A. Crowell, and C. Leighton, Phys. Rev. B 77, 014420 (2008).

${ }^{16}$ M. Fecioru-Morariu, J. Wrona, C. Papusoi, and G. Güntherodt, Phys. Rev. B 77, 054441 (2008).

${ }^{17}$ Z. Shi, X. P. Qiu, S. M. Zhou, X. J. Bai, and J. Du, Appl. Phys. Lett. 93, 222504 (2008).

${ }^{18}$ P. Y. Yang, C. Song, F. Zeng, and F. Pan, Appl. Phys. Lett. 92, 243113 (2008).

${ }^{19}$ X. P. Qiu, D. Z. Yang, S. M. Zhou, R. Chantrell, K. O'Grady, U. Nowak, J. Du, X. J. Bai, and L. Sun, Phys. Rev. Lett. 101, 147207 (2008).

${ }^{20}$ E. Jiménez, J. Camarero, J. Sort, J. Nogués, N. Mikuszeit, J. M. GarcíaMartín, A. Hoffmann, B. Dieny, and R. Miranda, Phys. Rev. B 80, 014415 (2009).

${ }^{21}$ M. D. Stiles and R. D. McMichael, Phys. Rev. B 59, 3722 (1999).

${ }^{22}$ D. Choo, R. W. Chantrell, R Lamberton, A Johnston, and K. O'Grady, J. Appl. Phys. 101, 09E521 (2007). 